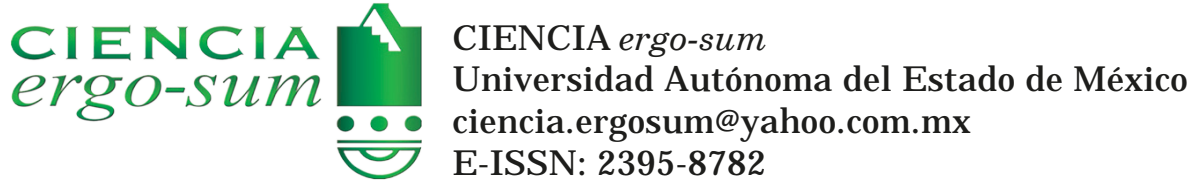

\title{
Propagación de ondas no lineales oscuras en fibras ópticas
}

\author{
Consuelo-Arriaga, Leonardo; Dávila Dávila, J osé Manuel; Agüero Granados, Máximo Augusto \\ Propagación de ondas no lineales oscuras en fibras ópticas \\ CIENCIA ergo-sum, vol. 28, núm. 3, noviembre 2021-febrero 2022 | e134 \\ Universidad Autónoma del Estado de México, México
}

Esta obra está bajo una Licencia Creative Commons Atribución-NoComercial-SinDerivar 4.0 Internacional .

Consuel o-Arriaga, L., Dávila Dávila, J . M. y Agüero Granados, M. A. (2021). Propagación de ondas no lineales oscuras en fibras ópticas. CIE NCIA ergo-sum, 28(3). https://doi.org/10.30878/ces.v28n3a6 


\title{
Propagación de ondas no lineales oscuras en fibras ópticas
}

\section{Propagation of dark nonlinear waves in optical fibers}

\author{
Leonardo Consuelo-Arriaga \\ Universidad Autónoma del Estado de México, México \\ leo.fisico@hotmail.com \\ (D) http://orcid.org/0000-0002-4801-8029 \\ José Manuel Dávila Dávila \\ Universidad Autónoma del Estado de México, México \\ jmdavilad@uaemex.mx \\ (D) http://orcid.org/0000-0002-7122-7195 \\ Máximo Augusto Agüero Granados \\ Universidad Autónoma del Estado de México, México \\ maaguerog@uaemex.mx \\ (D) http://orcid.org/0000-0002-9861-8997
}

Recepción: 04 de diciembre de 2019

Aprobación: 17 de enero de 2020

\section{RESUMEN}

Se reporta la dinámica de ondas solitónicas no lineales en óptica. La dinámica de estas estructuras está gobernada por la ecuación no lineal general de Schrödinger. Se muestra el comportamiento tipo partículas debido a la interacción de la dispersión y de la no linealidad expresada en la dependencia no lineal del índice de refracción del medio óptico. Los solitones se propagan en una fibra óptica sin alterar su forma y velocidad y, por lo tanto, pueden considerarse como un medio natural para transmitir información o energía sin pérdidas considerables. Sus propiedades de estabilidad dependen de la velocidad de las ondas solitónicas, que en el caso de ondas oscuras no lineales o burbujas son subsónicas.

Palabras ClaVEs: solitones, ondas no lineales, ecuación no lineal de Schrödinger.

\begin{abstract}
The dynamics of solitary waves in optics is reported. The dynamics of these structures is governed by the nonlinear Schrödinger's general equation. Particle-like behavior is shown, due to the interaction of dispersion and nonlinearity expressed in the nonlinear dependence on the refractive index of the optical medium. The solitons obtained are propagated in an optical fiber without altering their shape and speed and therefore can be considered as a natural means to transmit information or energy without significant losses. Their stability properties depend on the speed of solitonic waves which, in the case of nonlinear dark waves, or bubbles are subsonic.
\end{abstract}

KEYWORDS: solitons, nonlinear waves, nonlinear Schrödinger equation.

\section{INTRODUCCIÓN}

Akira Hasegawa, del laboratorio Bell de la empresa AT\&T, propuso que se utilizaran solitones ópticos para mejorar el rendimiento de las transmisiones en medios de comunicación usando las redes ópticas de telecomunicaciones (Hasegawa y Tappert, 1973). Fue siete años después de la predicción teórica de Hasegawa y Tappert que la transmisión de un solitón se demostró experimentalmente con éxito en una fibra óptica por Mollenauer, Stolen y Gordon (1980). Al respecto, se expone en este artículo la teoría relativa al surgimiento de ondas solitónicas en óptica y sus principales características. 
En la década de los setenta la empresa Corning Glass Works reportó una fibra óptica con un nivel de pérdida de unos $10 \mathrm{~dB} / \mathrm{km}$ que hizo factible su empleo en cuestiones industriales (Agrawal, 2001). En 1988 Linn Mollenauer y su equipo del mismo laboratorio transmitieron a más de $4000 \mathrm{~km}$ usando el efecto Raman (nombrado en honor de un científico indio que describió un método para amplificar las señales en una fibra óptica). En 1989 fue instalado el primer cable de fibra óptica submarino en el océano Pacífico, TPC-3, que va de Japón a Estados Unidos, con una capacidad de $280 \mathrm{Mbit} / \mathrm{s}$ por par de fibra. En 1991, también en el laboratorio Bell, se logró transmitir solitones a más de $14000 \mathrm{~km}$ donde se utilizaron amplificadores de Erbio. Para 1999 ya se tenía registro de velocidades de transmisión de $160 \mathrm{Gbit} / \mathrm{s}$ e incluso de $1 \mathrm{Tbit} / \mathrm{s}$, lo cual fue posible gracias a los desarrollos tanto teóricos como tecnológicos de los pulsos no lineales llamados solitones. Thierry Georges, quien trabajaba en el centro de investigación y desarrollo de France Telecom, en conjunto con su equipo, combinó solitones de longitudes de ondas diferentes para realizar una transmisión a razón superior a 1 terabit por segundo $\left(10^{12}\right.$ bits/segundo).

En 2001 los solitones logran una aplicación tecnológica con el equipo de telecomunicaciones. Estos eran utilizados para control de tráfico real de señales sobre una red comercial (Mollenauer y Gordon, 2006; Hasegawa y Matsumoto, 2003; Agrawal, 2001). Básicamente, el medio para transmitir es un elemento a través del cual se envía la información del emisor al receptor. Por ejemplo, una radiodifusora envía la información vía ondas de radio (microondas) a través de la atmósfera; una estación de comunicaciones basada en fibra óptica lo hace por pulsos en el infrarrojo a través de la fibra óptica. El científico Charles Kao (Premio Nobel de Física en 2009) sugirió que fibras ópticas eficientes podrían competir en la transmisión de información.

En el ámbito de la óptica, las ecuaciones de Maxwell se usan para describir la propagación de ondas electromagnéticas a través de diferentes medios. Sin embargo, los materiales tales como las fibras ópticas son dispersivos, no lineales y no homogéneos, y el campo eléctrico de la misma onda manifiesta una respuesta no lineal en su constante dieléctrica. Para describir la propagación de ondas electromagnéticas a través de estos medios dispersivos, no lineales y no homogéneos, se usan las ecuaciones diferenciales no lineales. Estos estudios pertenecen al ámbito de óptica no lineal. En particular, el comportamiento de ondas electromagnéticas en medios ópticos de respuesta no lineal fue investigado mediante la ecuación no lineal de Schrödinger (NLSE), introducida en los trabajos de Chiao, Garmire y Townes (1964) y de Talanov (1964), donde demostraron la conexión de la NLSE con los fenómenos de autoenfoque (self-focusing) de ondas electromagnéticas. Se mostró que los "paquetes" de ondas electromagnéticas preservan su forma a manera de una envolvente o modulación lenta al propagarse en medios no lineales y dispersivos.

\section{Propagación de luz en fibras ópticas}

La propagación de la luz está gobernada por las ecuaciones de Maxwell:

$$
\begin{array}{ll}
\nabla \times E=-\frac{\partial B}{\partial t}, & \nabla \times H=J-\frac{\partial D}{\partial t} \\
\nabla \cdot \mathrm{D}=\rho_{f}, & \nabla \cdot B=0 .
\end{array}
$$

Las densidades de flujo eléctrico $D$ y del flujo magnético $B$ en un medio están dados por:

$$
\begin{aligned}
& D={ }_{\epsilon} E+P, \\
& B={ }_{\mu 0} H+M,
\end{aligned}
$$

donde $P$ es la polarización y $M$ es magnetización, $\epsilon 0$ y $\mu_{0}$ son los coeficientes de permitividad del espacio libre y permeabilidad magnética respectivamente. 
Cuando un pulso de luz incide en una fibra óptica, influye en el comportamiento inarmónico de la nube electrónica que rodea a las moléculas, causada por su componente del campo eléctrico. Por lo tanto, se modifica el índice de refracción de las fibras ópticas, es decir, ahora dependerán de la intensidad de campo eléctrico incidente. Este efecto lleva el nombre de efecto Kerr. Esta respuesta no lineal permite la reestructuración del índice de refracción de la siguiente forma:

$$
n\left(\omega,|E|^{2}\right)=n_{0}(\omega)+n_{2}\left(\omega,|E|^{2}\right)+n_{4}\left(\omega,|E|^{4}\right),
$$

donde $E$ representa el campo eléctrico de la luz incidente. La no linealidad de orden superior surge para conservar así el grado superior de los términos no lineales en la polarización (Akhmediev y Ankiewicz, 1997).

No hace mucho se reparaba poco en la propagación de haces ópticos en un medio cuya no linealidad era de quinto orden debido a que no se tenía conocimiento de las soluciones analíticas; en apariencia, daba la impresión de que no era posible encontrar algún material con no linealidad y un índice de refracción que sea superior a segundo orden. En la actualidad se han retomado estas investigaciones y ha revivido el interés en investigar no linealidades superiores (Akhmediev y Ankiewicz, 1997). Se ha podido demostrar experimentalmente que la susceptibilidad óptica de lentes dopados de sulfuro de cadmio $\left(C d S_{x} S_{1-x}\right)$ posee una susceptibilidad de quinto orden. También fue posible la demostración de la existencia de no linealidades de quinto orden cuando se hace incidir pulsos de femtosegundos intensos con $620 \mathrm{Nm}$ en un cristal transparente (Hasegawa, 2002; Akhmediev y Ankiewics, 1997; Agüero-Granados y Alvarado, 2000).

Usando la no linealidad del índice de refacción de la ec. (4) y las ecuaciones de Maxwell, ec. (1) de Maxwell bajo un análisis matemático riguroso, y considerando las condiciones de $a$ ) monocromaticidad del frente, $b$ ) modulaciones espaciales lentas y $c$ ) no linealidad de Kerr, en $1+1$ dimensiones se puede encontrar que el campo eléctrico de la luz satisface la siguiente ecuación diferencial no lineal de Schrödinger

$$
i \psi_{t}+\Omega \psi_{x x}-\xi_{1} \psi+\xi_{2}|\psi|^{2} \psi-\xi_{3}|\psi|^{4} \psi=0
$$

A esta ecuación se le conoce como ecuación no lineal cúbica quinta de Schrödinger (c-q NLSE). La $t$ representa a la coordenada longitudinal y $x$ por su lado será la coordenada trasversal. De acuerdo con el orden de cómo están representados los términos de la ecuación, el coeficiente $\xi_{1}$ se relaciona de manera importante con la velocidad de grupo. El parámetro $\Omega>0$ se relaciona con la dispersión normal y los coeficientes para los términos no lineales cúbico y quinto se expresan como $\xi_{2}=k_{0} n_{2} \alpha_{1}, \xi_{3}=k_{0} n_{4} \alpha_{2}, k_{0}=\omega_{0} / c$, siendo $\omega_{0}$ la frecuencia de la onda. Los parámetros $\alpha_{1}, \alpha_{2}$ son coeficientes de dispersión para los componentes radiales del campo en el régimen no lineal.

\section{ECUACIONES DE MOVIMIENTO DE LOS PUlsos EN FIBRAS ÓPTICAS}

Para poder integrar la ecuación cúbica quinta de Schrödinger es conveniente representarla de forma que se muestren los mínimos de la energía potencial que da origen a la ecuación. No es difícil de hacer esta transformación en la ec. (5), por lo que de ahora en adelante se estudiarán las soluciones de la ecuación siguiente (Agüero-Granados y Serkin, 2020):

$$
i \phi_{t}+\phi_{x x}+\left(|\phi|^{2}-\rho_{0}\right)\left(2 A+\rho_{0}-3|\phi|^{2}\right) \phi=0
$$

siendo $A$ el parámetro principal del modelo, donde 


$$
\frac{A}{\rho_{0}}=-2+\frac{3}{4} \frac{\xi_{3}^{2}}{\xi_{1} \xi_{5}}\left[1+\sqrt{1-4 \frac{\xi_{1} \xi_{5}}{\xi_{3}^{2}}}\right] .
$$

De la ec. (6) es posible que surjan estructuras complejas como ondas no lineales de tipo escalón, llamadas también tipo kink y de tipo oscuro o burbuja debido a que se ha reportado el surgimiento de estas estructuras en procesos físicos modelados por dicha ecuación. Esto es consecuencia de que el término de orden cúbico y el de orden quinto se compensan mutuamente (Yang, 2010). Al respecto, se tratará de encontrar estas dos soluciones para la ec. (6) utilizando el ansatz:

$$
\varphi(x, t)=\sqrt{\rho(x, t)} e^{i \theta(x, t)}=\sqrt{\rho(\xi)} e^{i \theta(\xi)},
$$

y usando las soluciones de onda viajera: $\varphi(x, t)=\varphi(\xi)$, donde $\xi=x-v t$, la c-q NLSE se divide en dos ecuaciones:

$$
\begin{gathered}
\frac{\rho^{\prime \prime}}{2 \rho}-\frac{\rho^{\prime 2}}{4 \rho^{2}}-\theta_{\xi}(\theta \xi-v)-3(\rho-1)(\rho-\beta)=0, \\
\frac{v}{2 \rho}(\rho-\beta)=\theta_{\xi},
\end{gathered}
$$

$\operatorname{con} \beta=(2 A+1) / 3$.

Ahora, el enfoque está en las soluciones especiales tipo burbujas. Reemplazando la ec. (8) en la ec. (9) y denotando

$$
r=\rho-\beta, \quad a=\left[6 \beta(\beta-1)-v^{2}\right] / 4=\left(v \frac{2}{s} v^{2}\right) / 4,
$$

después puede encontrarse que las ecuaciones anteriores se reducen a la siguiente expresión para obtener soluciones:

$$
\pm\left(\xi-\xi_{0}\right)=\int \frac{1}{r \sqrt{r^{2}+(5 A-2) r / 3+a}} d r .
$$

Resolviendo la integral anterior, se obtiene la solución solitónica para $1<A<4$.

$$
\varphi_{b}=e^{i \theta} \frac{\sqrt{(4-\mathrm{A}) / 3} \operatorname{Cosh}(y)}{\sqrt{1+(4-\mathrm{A}) /(2 \mathrm{~A}+1) \operatorname{Sinh}^{2}(y)}},
$$

con $y=\left(v_{S} / 2\right)\left(\xi-\xi_{0}\right)$. La condición para que existan soluciones solitónicas es $v_{S}-v>0$, donde $v_{S}$ representa la velocidad del sonido. De la ec. (11) se ve que $\varphi_{b}$ es una función par de la variable (gráfica 1 ).

Esta solución describe una burbuja en el condensado. La amplitud de esta burbuja depende del valor de 4-A. Cuanto más pequeño es el valor de este parámetro, más pronunciado es el enrarecimiento de la burbuja. 


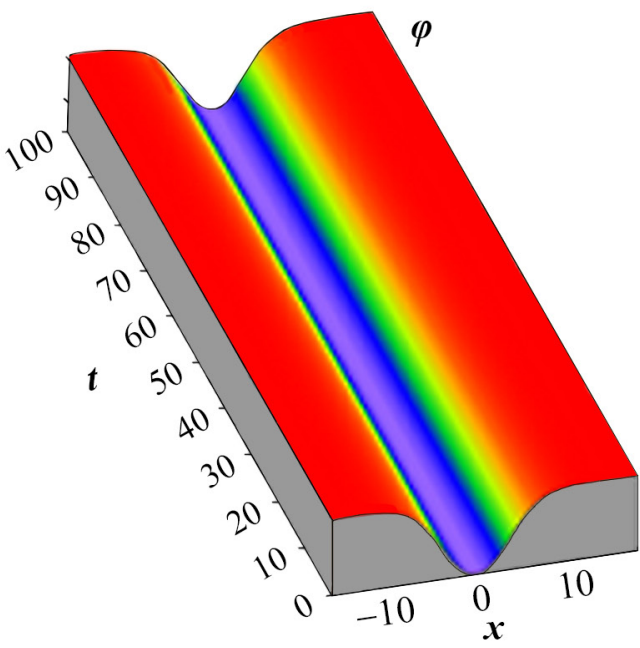

GRÁFICA 1

Solución tipo solitón oscuro que se propaga en un fondo de luz constante con $A=3.5$, obtenido al usar la ecuación (11)

Fuente: elaboración propia.

Una vez obtenida la solución solitónica, se calcularon las otras integrales de movimiento como el número de partículas $N$ y la energía $E$ para solitones tipo burbujas (b) y tipo kink (k).

$$
\begin{gathered}
N_{b}=-\operatorname{Arc} \operatorname{Cosh}\left\{\frac{b}{\sqrt{b^{2}-4 a}}\right\}, \\
E_{b}=\sqrt{a}(A / 2+1)+\left[A+\left(v^{2}-A^{2}\right) / 4\right] N_{b},
\end{gathered}
$$

$\operatorname{con} b=(5 A-2) / 3$.

Estas soluciones son huecos de intensidad en un fondo continuo de radiación electromagnética.

\section{Ondas en el límite de PeQueñas amplitudes}

Para el caso límite de pequeñas amplitudes, se deriva la ecuación no lineal de Korteweg y de Vries (KdV). Se consideran sólo ondas transónicas, es decir, las que son débilmente dependientes de coordenadas transversales. Para este propósito, se emplea la teoría de perturbación reductiva. Se consideran las nuevas variables definidas por $\xi=\varepsilon^{1 / 2}\left(x-v_{S} t\right), \tau=\varepsilon^{3 / 2} t$, donde $\varepsilon$ es un parámetro pequeño. A continuación, se expande el campo $\rho$ y $W$ $=\partial_{x} \theta$, en términos de potencias de $\varepsilon$ cerca del condensado:

$$
\rho=\beta, W=0, \rho=\beta+\epsilon \rho_{1}+\epsilon^{2} \rho_{2}+\cdots, W=\varepsilon W_{1}+\epsilon^{2} W_{2}+\cdots
$$

En el sistema de la ec. (9), para el primer orden de magnitud, se produce la ecuación de KdV:

$$
\partial_{\sigma} \rho_{1}-\partial_{\eta}^{3} \rho_{1}+3 \partial \eta \rho_{1}^{2}=0 \text {. }
$$


Con las relaciones entre las variables $\xi=(5 \beta-3)^{-1 / 2} \eta, \tau=2 v_{s}(5 \beta-3)^{-3 / 2} \sigma$. La solución expresada en términos de las variables originales toma la forma:

$$
\sqrt{\rho}=\left\{\beta-\frac{2 b}{5 \beta-3} \operatorname{Sech}^{2}\left[\sqrt{b}\left(x-v_{s} t+2 b /\left(v_{S}\right) t-x_{0}\right)\right]\right\}^{-1 / 2},
$$

con $4 b=g(5 \beta-3)>0$, siendo $g$ la velocidad del solitón. Esta solución se mueve en el condensado con una velocidad cercana, pero menor que la velocidad del sonido. Esta solución describe una onda localizada de enrarecimiento de campo y puede ser llamada también solitón burbuja. Para dimensiones mayores que 1, por ejemplo para $D=2$, la ec. (15) se transforma en la ecuación de Kadomtsev-Petviashvili (KP). La importancia de la "jerarquía” de KP se debe a su conexión con la gravedad 2D acoplada a la materia (Zakharov et al., 1984).

\section{Estabilidad de la soluCión SOlitónica OSCURA}

Como es conocido, uno de los criterios para determinar si la solución oscura es o no estable se basa en el uso de la integral de movimiento llamado momento renormalizado (Pelinovsky, Kivshar y Afanasiev, 1996). Este concepto surge debido a la divergencia que presenta el momento lineal clásico cuando se aplican condiciones de frontera no trivial o de tipo condensado. Por lo tanto, es necesario introducir las invariantes renormalizadas. Para este efecto, calculamos el momento renormalizado de la solución solitónica de la siguiente manera:

$$
P_{b}=-v \int_{-\infty}^{+\infty} \frac{\left(\Phi^{2}-\beta\right)^{2}}{\Phi^{2}} d \xi
$$

donde

$$
\Phi^{2}=\beta \frac{\operatorname{Cosh}^{2}(y)}{\gamma+\operatorname{Sinh}^{2}(y)}
$$

con $y=\frac{1}{2} \sqrt{v_{s}^{2}-v^{2}} \xi$, donde $v_{s}$ es la velocidad del sonido y $\xi=x-v t, \gamma=(2 A+1) /(4-A)$ y $\beta=(2 A+1) / 3$.

La solución solitónica oscura será estable si:

$$
\frac{\partial P_{b}}{\partial v}<0
$$

Para el caso de la ec. (16), que es la solución oscura o burbuja solitónica, la expresión para el momento renormalizado está dado por:

$$
P_{b}=-v \frac{4 \beta}{\sqrt{v_{s}^{2}-v^{2}}}\left\{\frac{(1+\gamma)}{\sqrt{\gamma(\gamma-1)}} \operatorname{Arc} \operatorname{Tanh} \sqrt{1-1 / \gamma}+\gamma-1\right\}
$$

Aplicando ahora el criterio de estabilidad de la ec. (19), se puede inferir que los solitones oscuros, cuya forma analítica se representa por la ec. (20), serán estables si el rango de existencia del parámetro principal se encuentra acotado por $1<A<4$, que exactamente coincide con el rango paramétrico de existencia de las soluciones oscuras para la ecuación no lineal de Schrödinger y sus velocidades no pueden sobrepasar la velocidad del sonido, es decir $v<v_{s}$, lo cual implica que su velocidad crítica es $v_{c r}=6 \beta(\beta-1)$. 


\section{Conclusiones}

Las fluctuaciones alrededor del condensado podrían causar la aparición de solitones tipo escalón o kink y pasar a través de un vacío inestable a otro vacío estable y simétrico. Si el kink es muy pequeño, lo que se gana en energía volumétrica para formarse en una región de vacío verdadero podría no ser suficiente para compensar la pérdida de energía en la superficie. Por lo tanto, es posible la aparición profusa de solitones grises o burbujas si perturbamos levemente el vacío degenerado relativo del potencial de interacción. En este caso las mutuas aniquilaciones y/o creaciones entre solitones oscuros podrían tener efecto, por lo que se observaría la creación y aniquilación de solitones oscuros en el fenómeno no lineal investigado. Este comportamiento es muy semejante al que muestra durante su interacción de partículas elementales. Para el caso de pulsos o solitones oscuros ya se han reportado su aparición en experimentos reales por ejemplo en Nakazawa y Suzuki (1995), que durante el proceso de interacción de la luz incidente y el medio de una fibra óptica es posible la formación de huecos de intensidad de luz viajera, en los cuales se podrían codificar alguna información importante y transmitirlo a largas distancias. En conclusión, de los resultados analíticos obtenidos se podría deducir que es posible la aparición de pulsos oscuros o solitones grises viajeros que se muestran como la ausencia de luz en el fondo de una onda de luz, los cuales son estables durante su traslado a lo largo de fibras ópticas. Esto ocurre siempre y cuando su velocidad no rebase la que posee el sonido en el medio porque, de no ser así, el pulso solitónico se tornaría inestable y desaparecería. El parámetro principal $A$ relacionado con el índice de refracción del medio, en la ecuación cúbica quinta de Schrödinger está restringido a la región de existencia de los solitones oscuros: $1<A<4$. Experimentalmente, se observa como una región oscura dentro de un pulso óptico normal.

\section{Prospectiva}

La existencia de solitones oscuros, huecos de campos o burbujas espacio temporales en diferentes fenómenos físicos es un hecho demostrable tanto teórico como experimental. En cuanto a los resultados teóricos, en este artículo se presenta el caso simple cuando se tiene una sola solución solitónica oscura o burbuja, esto es, cuando la componente eléctrica de la luz se propaga en una fibra óptica y crea zonas de sombra central

Para el caso de pulsos o solitones oscuros ya se han reportado su aparición en experimentos reales, por ejemplo en Nakazawa y Suzuki (1995), que durante el proceso de interacción de la luz incidente y el medio de una fibra óptica es posible la formación de huecos de intensidad de luz viajeras, en donde se podría codificar alguna información importante y transmitirla a largas distancias. Estas soluciones son menos sensibles al ruido y a la pérdida en fibras ópticas que las otras soluciones como las brillantes y, por ende, son más promisorias para usarse en comunicaciones ópticas como portadores de información a largas distancias siempre y cuando éstas se mantengan estables porque si no lo son, cualquier perturbación exterior al sistema de propagación destruiría la configuración inicial del pulso y de esa manera truncar la propagación del pulso oscuro. Por lo tanto, es importante analizar su estabilidad correspondiente.

Para el caso de una sola onda solitónica, en esta contribución se demuestra que esta estructura se mantiene estable cuando su velocidad de transmisión no rebasa a la velocidad del sonido. Por otro lado, la versión integrable de la ecuación cúbica quinta de Schrödinger en las inmediaciones de un mínimo local del potencial, que es la ecuación de Boussinesq mejorada (Agüero-Granados y Alvarado, 2000) soporta n-soluciones solitónicas. Por consiguiente, es importante analizar a posteriori la estabilidad cuando en la escena de la fibra óptica hacen su aparición dos o más pulsos ópticos solitónicos. Para esta nueva versión se aplicarían los criterios basados en el cambio de momentos renormalizados respecto al tiempo. 


\section{Agradecimientos}

Se agradecen los comentarios de los árbitros de la revista que mejoraron sustancialmente el contenido del artículo.

\section{REFERENCIAS}

Agrawal, G. P. (2001). Nonlinear fiber optics. San Diego: Academic Press.

Agüero-Granados, M. A., \& Alvarado, R. O. (2000). Bright and singular solitons in the boussinesq like equation. Physica Scripta, 62, 232-237.

Agüero, G. M. A., \& Serkin N. V. (2020). Introducción a la teoría de solitones. México. EON.

Akhmediev, N. N., \& Ankiewicz, A. (1997). Solitons nonlinear pulses and beams. London: Charpman \& Hall.

Chiao, R. Y., Garmire, E., \& Townes, C. H. (1964). Self-trapping of optical beam. Physical Review Letters, 13, 479-482.

Hasegawa, A., \& Matsumoto, M. (2003). Optical solitons in fibers. Berlin: Springer-Verlag.

Hasegawa, A., \& Tappert, F. D. (1973). Transmission of stationary nonlinear optical pulses in dispersive dielectric fibers. I. Anomalous dispersion. Applied Physics Letters, 23, 142-144.

Mollenauer, L. F., \& Gordon, J. P. (2006). Solitons in optical fibers. Boston: Academic Press.

Mollenauer, L. F., Stolen, R. H., \& Gordon, J. P. (1980). Experimental observation of

picosecond pulse narrowing and solitons in optical fibers. Physical Review Letters, 45, 1095.

Nakazawa, M., \& Suzuki, K. (1995). 10 gbits pseudorandom dark soliton datstransmission sobre $1200 \mathrm{~km}$. Electronics Letters, 31, 1084-1995.

Pelinovsky, D. E., Kivshar, Y. S., \& Afanasjev, V. V. (1996). Instability-induced dynamics of dark solitons. Physical Review E, 54, 2015-2032.

Talanov, V. I. (1964). Self-focusing of electromagnetic waves in nonlinear media. Izv. Vyss hikh. Uchebn. Zavedenii, Radiofiz, 7, 564.

Yang, J. (2010). Nonlinear waves in integrable and nonintegrable. USA: Systems, society for industrial and applied mathematics.

Zakharov, V., Manakov, S. V., Novikov, S. P., \& Pitaevsk, L. P. (1984). Theory of Solitons. New York: Plenum.

CC BY-NC-ND 